\title{
LA INFERENCIA CUANTIFICADA EN LA LÓGICA MEXICANA DEL SIGLO XVI
}

WALTER REDMOND

Austin, TeXas

A medida que los pensadores escolásticos trataban las complejidades de la inferencia, extendieron los límites del esquema sujeto-predicado. Describimos aquí el análisis de argumentos cuyas oraciones van más allá de este esquema en un texto de lógica de 1554 publicado en México y en España, el Recognitio summularum de fray Alonso de la Vera Cruz (1504-1584). ${ }^{1}$ Él y sus colegas adoptaron una aproximación extensional de la lógica donde la relación de identidad tenía un lugar central. Utilizaremos un lenguaje simbólico especialmente diseñado para ilustrar este sistema. ${ }^{2}$

Después de revisar algunas ideas de fray Alonso sobre la naturaleza del argumento (1), introducimos el simbolismo extensional y mostramos cómo puede desarrollarse la inferencia sobre la base de la relación de identidad, (2). Luego analizamos dos clases de inferencia que van más allá del esquema sujeto-predicado: los argumentos que contienen términos "oblicuos", ed aquellos casos latinos distintos del nominativo (3), y argumentos que

1 La silogística, especialmente los capítulos 1 y 14. Citamos página y columna (A o B) de la edición salmantina de 1573, pp. 94-121, en 20 capítulos. Alonso escribió una segunda obra sobre lógica, la Dialectica resolutio.

2 Para la lógica extensional escolástica véanse Redmond, "Extensional Interpretation of General Sentences in Sixteenth-Century Logic", Crítica, UNAM, vol. 13, no. 39, diciembre de 1981, pp. 45-73; "La lógica de las unidades divididas" y "La lógica de la existencia", capítulos 4 y 5 de Pensamiento y realidad en Fray Alonso de la Vera Cruz (escrito en colaboración con Mauricio Beuchot), UNAM, México, 1987; "Relations and 16th-Century Mexican Logic, Crítica, vol. 22, no. 65, agosto de 1990, pp. 23-41, abril 1983; "Modal Logic in Sixteenth-Century Mexico", Crítica, vol. 15, no. 43, abril de 1983, pp. 31-50; "Relaciones y unidades complejas en la lógica de fray Alonso de la Vera Cruz" y "'Reglas y condiciones': fray Alonso superando el marco sujeto-predicado", capítulos 3 y 4 de La teoría de la argumentación en el México colonial (en colaboración con Mauricio Beuchot), uNAM, México, 1995 y "Friar Alonso on The Logic of God", Vivarium, vol. 32, no. 3, 1994, pp. 227-260. 
contienen más de tres términos (4). Finalmente, examinamos la semántica de las oraciones oblicuas (5). Se anexa una lista de abreviaturas lógicas.

\section{Algunos puntos básicos}

\subsection{Argumentos no silogísticos}

La clase de argumentación que analizaremos aquí involucra la cuantificación (las nociones de "todo" y "alguno") y fue desarrollada por los lógicos de la época en el contexto de la silogística aristotélica. Sin embargo, en su teoría de la argumentación no se limitaron al silogismo. Alonso explica (116 B) que los manuales de lógica

generalmente trataron las formas oracionales del argumento, como cuando argumentamos en un condicional y de su antecedente a su consecuente. ${ }^{3}$

Hoy hablamos de la lógica proposicional, donde la inferencia se basa en reglas relacionadas a las conectivas " $y$ ", "o", "si", etc. (como en el argumento en 1.3. de abajo).

\subsection{Formalismo}

Alonso distinguió, al comienzo de su silogística (95 A), entre la validez de un argumento y el valor de verdad de sus oraciones, ed del hecho de que sus oraciones sean verdaderas o falsas. La lógica estudia:

el silogismo formal, donde la verdad o falsedad de las premisas y la conclusión no se consideran, en tanto se mantenga el modo y la figura correcta, porque el silogismo es formalmente válido incluso cuando sus premisas y conclusión son falsas. ${ }^{4}$

La lógica no toma en cuenta el contenido, pero sí la afirmación y la negación, dice Alonso, y llama a este silogismo "verdadero en su forma, si no en su contenido":

todo hombre es un burro

todo caballo es un hombre

3 Etiam solent poni modi syllogizandi in hypotheticis, ut si quis argumentetur a tota conditionali cum positione antecedentis ad positionem consequentis. Alonso nos remite a su propio tratamiento de la inferencia oracional, dando este ejemplo: si todo hombre corre, un animal corre/ un hombre corre/ luego: un animal corre.

${ }^{4}$ Et hic tractatus de syllogismo formali, qui abstrahit a veritate vel falsitate propositionum et conclusionis, dummodo fiat syllogismus secundum debitum modum et figuram, quia est validus de forma etiamsi praemissae et conclusio sint falsae. 
luego

todo caballo es un burro

Considera este argumento como una implicación donde tanto el "antecedente" (se refiere a la conjunción de las premisas) como el "consecuente" (la conclusión) son falsos. La inferencia es lógicamente válida pues cuando el antecedente y el consecuente son falsos, la implicación como un todo es verdadera (una implicación es falsa sólo en caso de que el antecedente sea verdadero y el consecuente falso).

\subsection{Dos tipos de modalidad}

Las reglas generales de la silogística tienen dos sentidos, dice Alonso (95 B), y corresponden a dos obras de Aristóteles. En los Analíticos posteriores la cuantificación se sobreentiende, pues en la "materia natural" estudiada en la obra los términos están necesariamente engarzados. Por ejemplo la oración "un hombre tiene conocimiento sensible"; puesto que nada que carezca de conocimiento sensible puede ser humano, es inferencialmente equivalente a "todo hombre tiene conocimiento sensible". Pero la lógica formal de los Primeros analíticos exige que se exprese la cuantificación. Porque cuando decimos que una conclusión se sigue "necesariamente" de las premisas, dice Alonso (95 B), estamos hablando de la necesidad "de la inferencia", no "del consecuente", y:

una vez que se exponen y admiten las premisas, debe también ser verdadero que algo se sigue, no que esta cosa es necesaria. ${ }^{5}$

Y en este ejemplo:

\section{necesariamente, si uno corre uno se mueve}

la expresión modal no se aplica a "uno se mueve" sino que quiere decir:

dado el antecedente de que uno corre, la inferencia de que uno se mueve es válida. ${ }^{6}$

Hoy diríamos: no: $\square \mathrm{M}$ sino: $\square[[[\mathrm{C} \supset \mathrm{M}] \& \mathrm{C}] \supset \mathrm{M}]$. Este argumento, por supuesto, no es un silogismo, pero involucra reglas de la lógica proposicional.

5 oportet praemissis positis et concessis, etiam sit verum quod aliquid sequitur, non tamen dicit necessarium esse.

6 quod posito antecedenti quod currat, sequitur in bona consequentia quod moveatur. 
2. Lógica extensional de la identidad

\subsection{Simbolismo}

Los escolásticos de los tiempos de Alonso distinguieron dos tipos de términos para las cosas individuales: nombres propios como "Pedro" y "términos vagos" como "este hombre", refiriéndonos, digamos, a Pedro. Los últimos combinan un término general ("hombre") con un demostrativo ("este", "aquel", "aqueste"). Usaremos letras minúsculas para términos individuales, por ejemplo, " $p$ " para Pedro y " $h$ " con un subíndice numérico para hombre; los subíndices numéricos nos servirán como demostrativos, así el término vago "este hombre" se simboliza como $h_{1}$ : "hombre número-uno", Pedro. Pedro se llama "este hombre" si y sólo si tiene la propiedad de ser un ser humano.

Cuando decimos que "Pedro es este hombre" la relación entre los términos es de identidad, el sentido es que Pedro es idéntico a este hombre, o que Pedro tiene dos nombres: "Pedro" y "este hombre". Para expresar simbólicamente esta relación, simplemente juntamos los dos términos: $p h_{1}$. Para negar oraciones usaré la diagonal: si $h_{2}$ se refiere, digamos, a Pablo, entonces $p / h_{2}$ "Pedro no es ese hombre", refiriéndonos a Pablo.

Los escoláticos consideraban básica una oración de identidad como $p h_{1}$ que sólo contiene términos singulares. Para formar una oración general como "Pedro es (algún) hombre" necesitamos la cuantificación. Usaré corchetes para "(por lo menos) un hombre", $[h]$ y paréntesis para "todo hombre", (h). Corchetes y paréntesis funcionan como cuantificadores universal y particular respectivamente. Así $p[h]$ ("Pedro es un hombre") y $p /(h)$ ("Pedro no es todo hombre") son oraciones verdaderas.

Los lógicos escolásticos definieron la cuantificación en términos de operaciones complementarias: "descenso" y "ascenso" (o "inducción"). En el descenso las oraciones generales se analizan en términos de identidades unidas por la " $o$ " $(v)$ :
$1 \mid p[h]$
hipótesis
$2 \mid p h_{1} v p h_{2} v \ldots v p h_{n}$
descenso disyuntivo del paso 1

Si Pedro es hombre, entonces es o este, o ese o aquel etc. Esta clase de descenso se llama "disyuntivo" pues su análisis es una disyunción. El segundo paso (que se lee "Pedro es el hombre-número-1 o es el hombre-número-2 o ... es el hombre-número-n" es verdadera aunque $p h_{1}$ sea la única identidad verdadera, porque si uno de los disyuntos es verdadero, la disyunción total lo es.

La operación conversa es el ascenso disyuntivo: 
$\begin{array}{ll}1 \mid p h_{1} v p h_{2} v \ldots p h_{n} & \text { hipótesis } \\ \vdash & \\ 2 \mid p[h] & \text { ascenso disyuntivo } 1\end{array}$

El descenso y ascenso conjuntivos llevan " $y$ " ' \&) en lugar de "o". Que Pedro sea todo animal $p(a)$ es falso puede verse claramente por su análisis:
$1 \mid p(a)$
hipótesis
$2 \mid p a_{1} \& p a_{2} \& \ldots \& p a_{n}$
descenso conjuntivo en 1

pues, suponiendo que " $a_{1}$ " es un nombre correcto, $p a_{1}$ es el único conyunto verdadero y una conjunción es falsa a menos que todos sus conyuntos sean verdaderos; se discute aquí, dicho sea de paso, el problema de los conjuntos no denumerables.

Los lógicos escolásticos usaron este procedimiento para construir un sistema general de lógica de predicados. Por ejemplo, el análisis del enunciado general "todo hombre es animal", expresado como "todo hombre es algún animal" es (las reglas están abreviadas, consultar el apéndice):
$1 \mid(h)[a]$
h
$2 \mid h_{1}[a] \& h_{2}[a] \& h_{n}[a]$
dc 1
3 | $h_{1}[a]$
ec 2
$4 \mid h_{2}[a]$
ec 2
...
$5 \mid h_{n}[a]$
ec 2
$6 \mid h_{1} a_{1} v h_{1} a_{2} v \ldots v h_{1} a_{o}$
dd 3
$7 \mid h_{2} a_{1} v h_{2} a_{2} v \ldots v h_{2} a_{0}$
dd 4
|..
$8 \mid h_{n} a_{1} v h_{n} a_{2} v \ldots v h_{n} a_{o}$
dd 5
$9 \mid\left\{h_{1} a_{1} v h_{1} a_{2} v \ldots v h_{1} a_{o}\right\} \&$
$h_{2} a_{1} v h_{2} a_{2} v \ldots v h_{2} a_{o} \& \ldots \&$$$
\left\{h_{n} a_{1} v h_{n} a_{2} v \ldots v h_{n} a_{o}\right\} \quad \text { ic } 6,7,8
$$

Para la validez general, los procesos de descenso y ascenso deben comenzar por la izquierda. Es fácil ver que el cuadrado normal de oposición va como sigue:

todo hombre es animal

(h) $[a]$

algún hombre es animal

$[h][a]$ ningún hombre es (algún) animal

$$
(h) /(a)
$$

algún hombre no es animal

$[h] /(a)$ 
De acuerdo con la lógica escolática extensional de la identidad, las oraciones generales se pueden reducir, mediante las operaciones del ascenso y descenso, a conjunciones y/o disyunciones de identidades con términos singulares; las oraciones generales son equivalentes a estas cadenas de identidades.

\subsection{Deducción por identidades}

Veamos cómo se puede establecer la validez de la inferencia cuantificada por medio de dicha reducción, con aplicación de una regla de identidad (la "regla-i"), también llamada ley de Leibniz, ley de la indiscernibilidad de lo idéntico, eliminación de la identidad (ei), etcétera:
$1 \mid \phi a$
h
$2 \mid a=b$
h
F
$3 \mid \phi b$
i, 1,2

Si dos "cosas" ( $a$ y $b$ ) son idénticas, entonces lo que se le atribuye a una $(\phi)$ se le atribuye a la otra."

Usaré el procedimiento (lo cual hasta donde yo sé, no fue llevado a cabo por los escolásticos) para examinar el análisis de Alonso. Podemos formalizar el siguiente silogismo sencillo:

todo hombre es animal

Pedro es hombre

- luego

Pedro es animal

de esta manera:

\begin{tabular}{l|ll}
1 & $(h)[a]$ & $\mathrm{h}$ \\
2 & $p[h]$ & $\mathrm{h}$ \\
& $\mid$ & \\
3 & $h_{1}[a] \& h_{2}[a] \& \ldots \& h_{n}[a]$ & $\mathrm{dc} 1$ \\
4 & $h_{1}[a]$ & ec 3 \\
5 & $h_{2}[a]$ & ec 3 \\
& $\ldots$ & ec 3 \\
6 & $h_{n}[a]$ & dd 2 \\
7 & $p h_{1} v p h_{2} v \ldots v p h_{n}$ &
\end{tabular}

7 Alonso explica varios aspectos de procedimientos análogos a las reglas para la transitividad de la identidad y ei en su tratado sobre los términos "divinos", De modo syllogizandi in terminis divinis, Salamanca, 1573, cap. 12, 112 A-115 A; véase Redmond, "Friar Alonso on The Logic of God". 


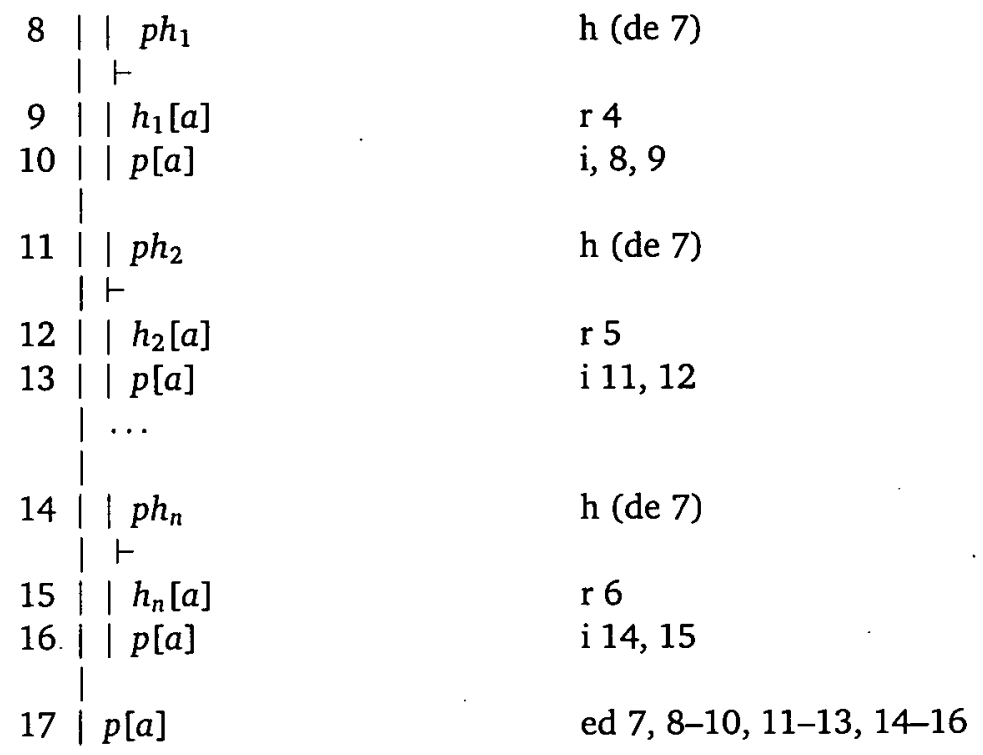

Nota que la operación clave es la regla-i en los pasos 10, 13 y 16.

No es necesario aplicar las reglas del descenso y ascenso al término a en el argumento de arriba, y la simplicidad se refleja en una prueba moderna:

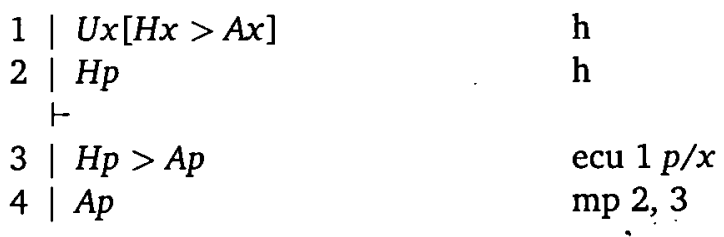

3. Términos oblicuos

Presentaremos primero la aproximación general de Alonso a los argumentos que contienen términos oblicuos (3.1.) y luego pasamos a considerar las dos reglas especiales que ofrece (3.2.). ${ }^{8}$

\subsection{Doctrina general}

3.10. Un primer ejemplo

He aquí un ejemplo del caso genitivo, Petri (de Pedro):

omnis hominis est asinus

8 Cap. 14 (116 A-117 A). 
Petrus est homo

ergo

Petri est asinus

(de todo hombre hay un burro - e.g., a cada hombre pertenece un burro; Pedro es hombre, luego: de Pedro hay un burro).$^{9}$ En este ejemplo, dice fray Alonso, el término medio es oblicuo sólo en la primera premisa; puede ser también oblicuo en la segunda e igual puede ser oblicuo el término mayor o el menor (aquellos que aparecen con el término medio en la primera y segunda premisas respectivamente).

\subsection{Análisis extensional}

Para representar los términos oblicuos necesitamos el signo " $\Re$ " para indicar cualquier relación en este contexto; aquí tenemos la relación del genitivo, es decir, de propiedad: $\Re p$ : "lo que es de Pedro". Nota el paralelismo con la prueba simple de arriba y cuyos términos están en caso nominativo.
$1 \mid \Re(h)[b]$
h
$2 \mid p[h]$
$\mathrm{h}$
$3 \mid \Re h_{1}[b] \& \Re h_{2}[b] \& \ldots \& \Re h_{n}[b]$ dc 1
$4 \mid \Re h_{1}[b]$
ec 3
$5 \mid \Re h_{2}[b]$
ec 3
$6 \ddot{\Re h}_{n}[b]$
ec 3
$7 \mid p h_{1} v p h_{2} v \ldots v p h_{n}$
dd 2
8||$p h_{1}$
h (de 7)
9||$\Re h_{1}[b]$
r 4
10||$\Re p[b]$
i 8,9
11||$p h_{2}$
h (de 7)
12||$\Re h_{2}[b]$
r 5
13 | $\Re p[b]$
i 11,12
I ...
14
$\mid p h$
h (de 7)
$1 \vdash$
9 La traducción sigue el orden regular (artificial) del latín. 


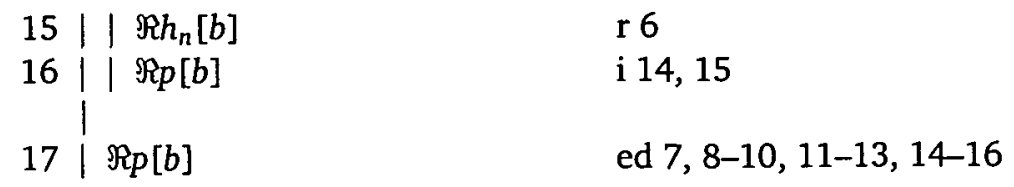

Una prueba en lógica actual corre paralela a este argumento (P: poseer, ser propietario de):

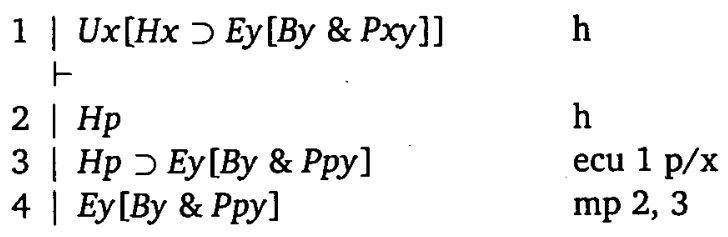

pero como "Ey[By \& Ppy]" no tiene que analizarse en detalle para llegar a una conclusión, podemos abreviarla como $\phi x$ y simplificar la prueba de esta manera:
$1 \mid U x[H x \supset \phi x]$
h
1
2 | $H p$
$\mathrm{h}$
$3 \mid M p \supset \phi x$
ecu $1 p / x$
$4 \mid \phi x$
mp 2,3

\subsection{Interpretación relacional}

Podemos ofrecer, dentro del contexto extensional, otra interpretación de los términos oblicuos como unidades complejas "complexivas, con sentido múltiple". ${ }^{10}$ La primera premisa del silogismo oblicuo en 3.11. sería, como si dijéramos, "todo hombre y un burro están genitivamente relacionados" y su conclusión "Pedro y un burro están genitivamente relacionados". La siguiente prueba es como la precedente (y como el silogismo sencillo de 2.2.) excepto en que se añade el paso 3a (g: "el par ordenado cuyos componentes están genitivamente relacionados", e.g. como propietario y propiedad): ${ }^{11}$

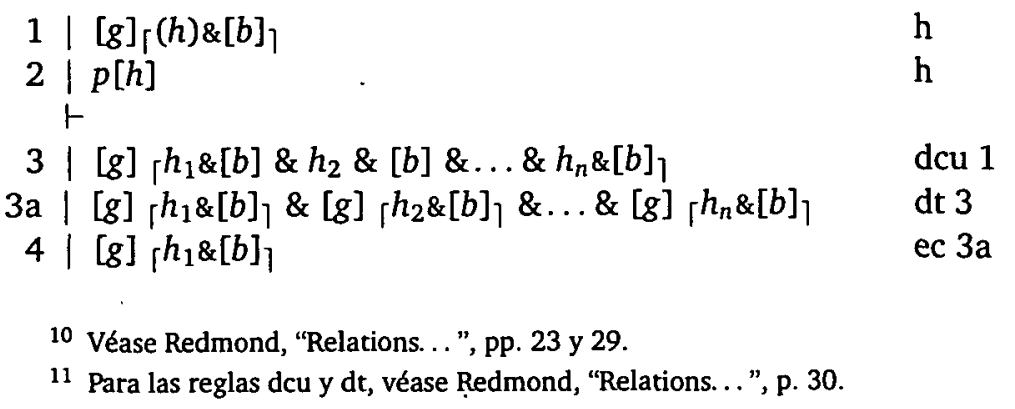




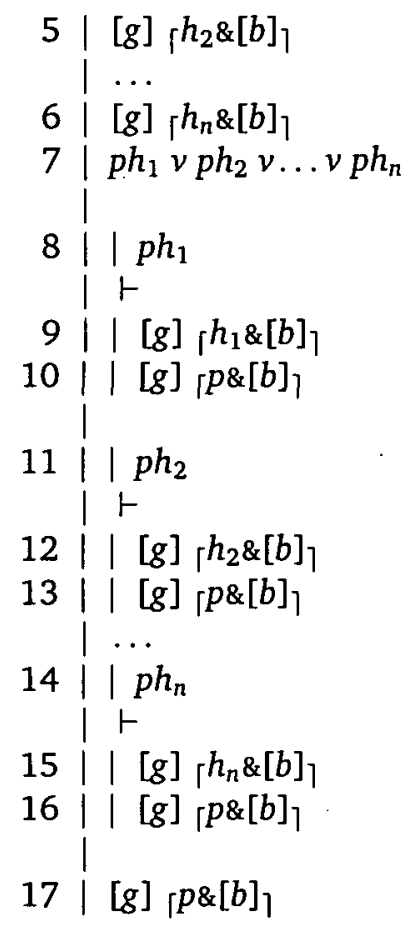

3.2. Dos reglas ec 3a

ec $3 a$

dd 2

h

r 4

i 8,9

h

r 5

i 11,12

$\mathrm{h}$

r 6

i 14,15

ed $7,8-10,11-13,14-16$

3.20. Necesidad de las reglas

Nota que en la prueba extensional arriba (3.11.) $\Re$ mantiene siempre la misma posición. ${ }^{12}$ Alonso dice $(116 \mathrm{~A})$ que si el término mayor o menor es oblicuo en una premisa, también debe serlo en la conclusión ${ }^{13}$-punto que será incorporado en la primera regla (3.21.) - y ofrece este ejemplo:

omne jumentum est hominis

omnis equus est jumentum

ergo

omnis equus est hominis

(todo jumento es de - pertenece a- un hombre; todo caballo es un jumento, luego: todo caballo es de un hombre).

El silogismo parece seguirse de acuerdo con las reglas ordinarias de la silogística:

12 Como los modales y otros operadores; véase Redmond, "Modal. ..", p. 34.

13 Si contingat oblique poni extremitatem aliquam, sic debet poni in conclusione. 
\begin{tabular}{l|ll}
1 & $(j) \Re[h]$ & $\mathrm{h}$ \\
2 & $(c)[j]$ & $\mathrm{h}$ \\
& $\vdash$ & \\
3 & $(c) \Re[h]$ & barbara 1,2
\end{tabular}

Pero dice Alonso (116 B) que para obtener inferencias correctas en esta clase de argumentos, además de observar las reglas de la silogística, debemos observar dos reglas especiales. ${ }^{14}$

\subsection{La primera regla}

La primera regla se aplica a:

todos los modos donde ambas premisas sean afirmativas, no importa a qué figura pertenezcan. ${ }^{15}$

El punto crucial es que una relación $\Re$ debe ocupar cierta posición en la estructura de la prueba (y en el proceso de ascenso/descenso).

Antes de dar la regla, veamos uno de los ejemplos de Alonso, el siguiente silogismo con un término oblicuo, el término medio:

quilibet homo est animal

omnis equus est hominis

ergo

omnis eqqus est animalis

non ... omnis equi est animal

(todo hombre es animal/ todo caballo es de algún hombre/ luego: todo caballo es de algún animal, no: de todo caballo hay algún animal). El argumento corresponde a este esquema:
$1 \mid(M)[J]$
$\mathrm{h}$
$2 \mid(N) \Re[M]$
h
1
$3 \mid(N) \Re[J]$
barbara 1,2

La conclusión no es, como dice: $\Re(N)[J]$

La regla expresa un requisito general y distingue dos casos de aplicación:

(1) cuando en una premisa el término mayor o menor es oblicuo y

14 Ad recte concludendum in istis vis intellectus attendenda potius quam ad syllogismorum regulas supra positas, licet possint poni saltem dua regulae.

15 In omnibus modis affirmativis ubi utraque praemissarum affirmativa est, cujusque sit figurae. 
(2) cuando en una premisa el término medio es oblicuo -en el ejemplo de arriba el término medio es oblicuo en la segunda premisa.

Si una premisa contiene un término oblicuo [paso 2 del esquema] la conclusión [3] también contendrá uno, de tal modo que:

[1] si un término mayor o menor es oblicuo en una premisa lo será también en la conclusión,

[2] si el término medio es oblicuo en una premisa [" $\Re \mathrm{M}$ " en 2] debemos asegurarnos de que el término que aparece con el término medio cuando éste es nominativo ["J" con "(M)" en 1] sea oblicuo en la conclusión [" $\Re$ $J^{\prime}$ '] en 3] y que el término que aparece con el término medio cuando éste es oblicuo ["N" en 2] sea nominativo en la conclusión ["N" en 3]. ${ }^{16}$

La regla se sigue, evidentemente, en el silogismo de arriba, y también en este ejemplo:

cujuslibet hominis est equus

Petrus est homo

ergo

Petris est equus

non...: Petrus est equi

(de todo hombre hay un caballo; Pedro es hombre, luego: de Pedro hay un caballo, no: Pedro es de un caballo), dando el esquema:

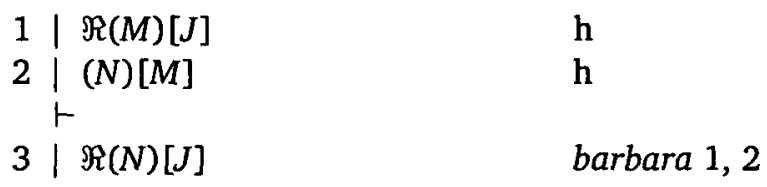

y la conclusión no es: (N) $\Re$ [J]. Alonso señala el lugar equivocado del término oblicuo y agrega:

la conclusión evidentemente no se sigue con "Petrus" en nominativo. ${ }^{17}$

Es interesante comparar esta regla con un pasaje que fray Alonso añadió (98B) a ediciones posteriores en un pasaje sobre defectos en la inferencia ${ }^{18}$

16 Si altera praemissarum est de obliquo et similiter conclusio erit, ita quod si extremitatum aliqua ponitur in obliquo in praemissis, et erit in conlusione. Si tamen medium ponatur in altera in obliquo, observandum ut extremitas quae ponitur cum medio in recto, ponatur in conlusione in obliquo, et quae ponitur cum medio in obliquo ponatur in conlusione in recto.

17 manifestum est conclusionem in recto non inferri.

18 Capítulos 2 y 3, pp. 97-98. 
El argumento es inválido, dice, "cuando el término mayor o menor están mezclados en la parte o en el todo", como en:

omne secundum inmediate sequitur primum omne tertium inmediate sequitur secundum ergo omne tertium inmediate sequitur primum ${ }^{19}$

(todo 2 inmediatamente sigue a 1; todo 3 inmediatamente sigue a 2, luego: cada 3 inmediatamente sigue a 1). El problema es que solamente

una parte del término mayor ("inmediatamente sigue a 1") se repite en la premisa menor, pero esta premisa debería ir así "todo 3 es un 2 ".

Si consideramos el segmento "un número que inmediatamente sigue al número n" como análogo a los términos oblicuos $(\Re n)$, tenemos el argumento y el esquema siguientes:

( (2) $\Re[1]$

(3) $\Re[2]$

$\vdash$

(3) $\Re[1]$

(M) $\Re[J]$
$(N) \Re[M]$
(N) $\Re[J]$.

Para resolverlo, dice Alonso, la menor debe convertirse en (3) [2] y el resultado es un silogismo barbara lógicamente válido (con premisa y conclusión falsas):

$(M) \Re[J]$
$(N)[M]$
$(N) \Re[J]$,

lo cual cumple con la primera parte de la regla de arriba. ${ }^{21}$

\subsection{La segunda regla}

La segunda regla se aplica solamente a argumentos negativos, pero antes de presentarla examinemos un silogismo negativo con un término oblicuo.

19 est permixtio extremitatum in parte vel in toto.

20 quoniam major extremitas, quae est hoc inmediate sequitur primum, replicatur secundum partem in minori, sed minor sic formetur: omne tertium est secundum.

21 Nota, sin embargo, que incluso cuando la relación $\Re$ "sigue" simplemente (sin el "inmediatamente"), el argumento es inválido sin otra premisa, puesto que no toda relación es transitiva. Donde $\phi$ nxrepresenta Ey[ny \& Sxy], $U x[2 y>\phi 1 x] \& U x[3 x>\phi 2 x]$ no implica. $U x[3 x>\phi 1 x]$. 
3.221. Argumentos negativos

Alonso ofrece (11 A) este ejemplo de un silogismo negativo cuyo término medio es un término oblicuo:

nullus equus est hominis

omnis asinus est hominis

igitur

nullus asinus est equus

(ningún caballo es de cualquier hombre/ todo burro es de un hombre/ luego: ningún burro es un caballo):
$1 \mid(c) / \Re(h)$
$\mathrm{h}$
$2 \mid(b) \Re[h]$
h
$3 \mid(b) /(c)$
cesare 1,2

Puede verse fácilmente su validez ( $\phi x$ es abreviación de $E y[H x \&$ Pyx]):
$1 \mid U x[C x>\sim \phi x]$
$\mathrm{h}$
$2 \mid U x[B x>\phi x]$
h
$3|x| \mid B x$
h
$4 \mid \begin{aligned} & 1 \\ & \mid B x>\phi x\end{aligned}$
r 2, ecu
5|| $\mid \phi x$
mp 3, 4
6|| $\mid C x>\sim \phi x$
r 1 , ecu
7|| $\mid \sim C x$
mt 5,6
8||$B x>\sim C x$
ii 3-7
$9 \mid U x[B x>\sim C x]$
icu 3-8

Parte de la prueba extensional iría así:
$1 \mid(c) / \Re(h)$
$2 \mid(b) \Re[h]$
h
h
$3 \mid c_{1} / \Re(h) \& c_{2} / \Re(h) \& \ldots \& c_{n} / \Re(h)$
dc 1
$4 \mid c_{1} / \Re(h)$
ec 3
$5 \mid c_{2} / \Re(h)$
ec 3
I $\ldots$
$6 \mid c_{n} / \Re(h)$
ec 3
$7 \mid b_{1} \Re[h] \& b_{2} \Re[h] \& \ldots \& b_{n} \Re[h]$
dc 2
$8 \mid b_{1} \Re[h]$
ec 7
$9 \mid b_{2} \Re[h]$
ec 7 


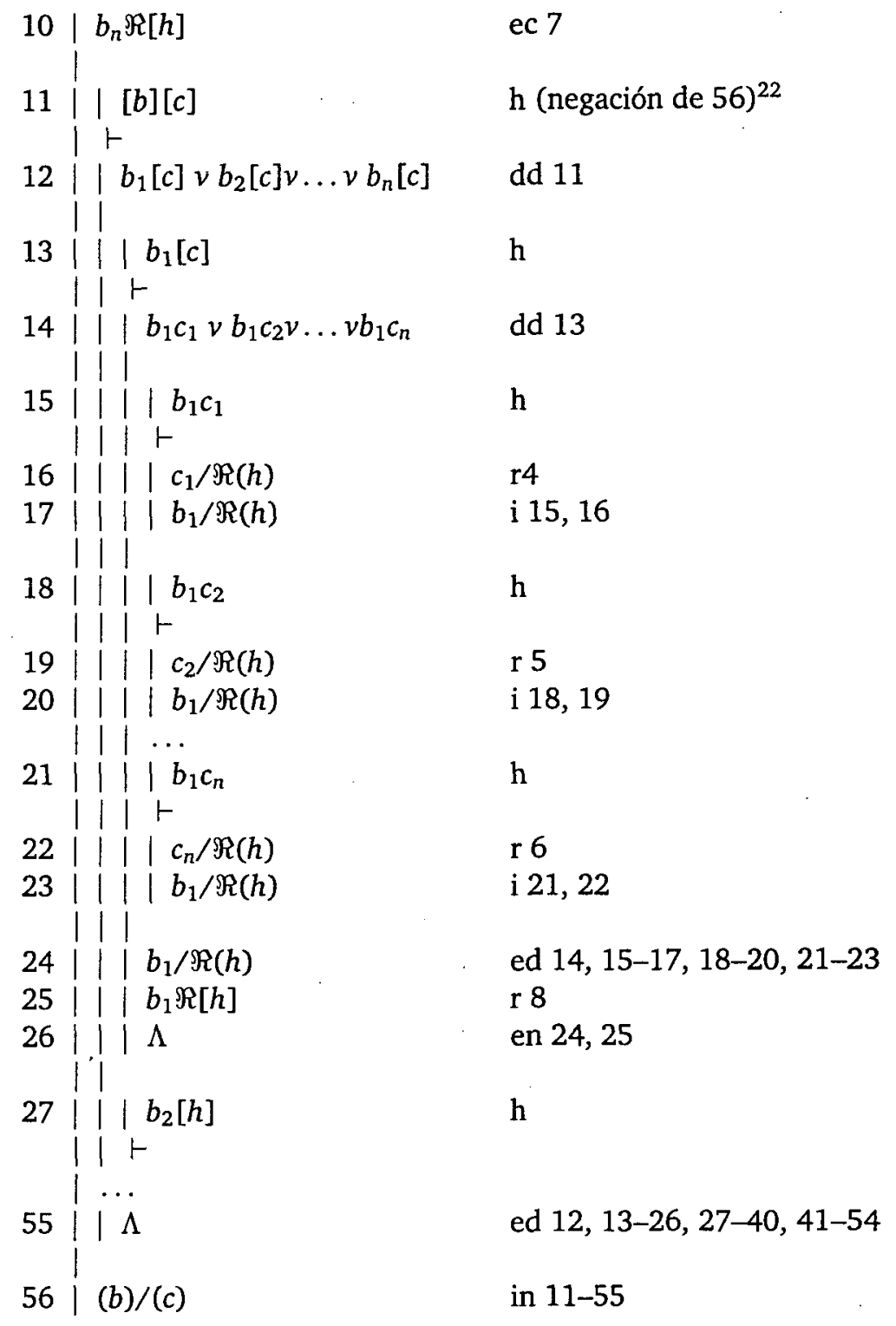

El signo $\Lambda(26,55)$ indica el absurdo al seguirse una contradicción.

3.222. La regla

La segunda regla también tiene dos partes:

22 Redmond, “Extensional. .. ", pp. 46-47. 
cuando un silogismo contiene una premisa con un término en nominativo y otra con un término oblicuo:

[1] si la premisa que contiene el término oblicuo es negativa, la conclusión será también negativa y contendrá un término oblicuo:

[2] pero si la premisa que contiene el término oblicuo es afirmativa y la que contiene el término en nominativo es negativa, la conclusión no tendrá la misma forma. ${ }^{23}$

Alonso ilustra la primera parte de la regla con dos silogismos. El primero:

nullus animalis est aeternitas

omnis homo est animal

ergo

nullius hominis est aeternitas

(de ningún animal hay eternidad/ todo hombre es animal/ luego: de ningún hombre hay eternidad). Aquí la primera premisa y la conclusión son negativas y contienen un término oblicuo. En el segundo ejemplo (en el modo festino) el término oblicuo aparece en dativo:

intellectus non est bruto

simia est brutum

ergo

simiae non est intellectus

no hay entendimiento para una bestia/ un mono es una bestia/ luego: para un mono no hay entendimiento).

La segunda parte de la regla apunta a bloquear inferencias como:

nullus homo est brutum

omnis equus est hominis

ergo

nullus equus est bruti

(ningún hombre es una bestia/ todo caballo es de un hombre/luego: ningún caballo es de una bestia). Incluso la siguiente interpretación resulta un argumento inválido:

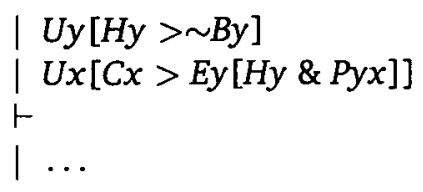

23 Quando syllogismus sit ex altera de recto et altera de obliquo, si negativa sit de obliquo et conclusio similiter; si vero affirmativa de obliquo et negativa de recto, non similiter concluditor. 
| $U x[C x>\sim E y[B y \& P y x]]$.

El hecho de que un hombre difiera de una bestia no quiere decir que ésta no pueda tener la misma relación hacia algo. ${ }^{24} \mathrm{El}$ segundo ejemplo tiene la misma forma (aunque contiene un nombre propio, "Dios"):

nullus homo est Deus

omne jumentum est hominis

ergo

nullum jumentum est Dei

(ningún hombre es Dios/ todo jumento es de un hombre/ luego: ningún jumento es de Dios) y es inválido por la misma razón básica. Este argumento paralelo es inválido:

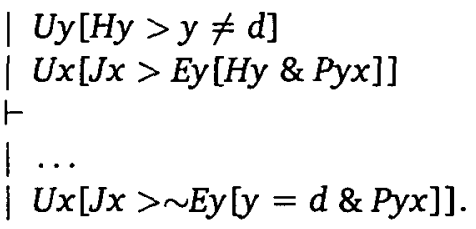

Pero, dice Alonso, las mismas premisas producen esta conclusión:

omne jumentum est alicujus quod non est Deus

(todo jumento es de algo que no es Dios). Esta prueba actual que contiene la nueva conclusión es válida:

\begin{tabular}{|c|c|c|}
\hline 1 & $U y[H y>y \neq d]$ & $\mathrm{h}$ \\
\hline 2 & $U x[J x>E y[H y \& B y x]]$ & $\mathrm{h}$ \\
\hline 3 & $x \mid \underset{\vdash}{\mid} J x$ & h \\
\hline 4 & $\mid J x>E y[$ Hy \& Pyx] & r 1 \\
\hline 5 & | Ey $[H y \& P y x]$ & $\mathrm{mp} 3,4$ \\
\hline 6 & $\left\{\begin{array}{l}y \mid H y \& P y x \\
\vdash\end{array}\right.$ & $\mathrm{h}$ \\
\hline 7 & $1 \mid \mathrm{Hy}$ & ec 6 \\
\hline 8 & | | Pyx & ec 6 \\
\hline 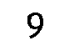 & ||$H y>y \neq d$ & r 1. ecu \\
\hline 10 & $y \neq d$ & $\mathrm{mp} 7,9$ \\
\hline 11 & ||$y \neq d \& P y x$ & ic 10,8 \\
\hline 12 & ||$E y[y \neq d \& P y x$ & ice 11 \\
\hline
\end{tabular}

24 La conclusión podría ser falsa (una yegua puede ser “de" un garañón, etcétera). 


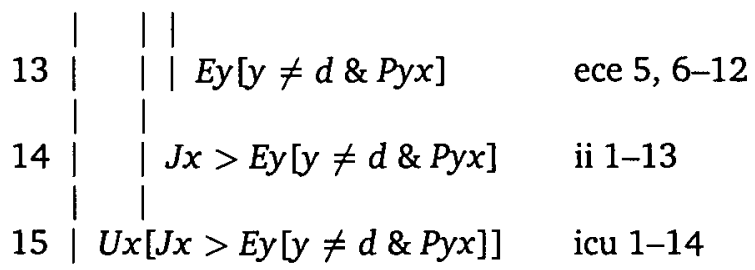

La conclusión difiere de la del silogismo inválido solamente en la posición del signo de la negación en el consecuente $(E y[\sim(y=d) \& P y x]$ en lugar de $\sim E y[y=d \& P y x])$.

Comparemos, usando el sistema extensional, las conclusiones $(j) / \Re(d)$ y (j) $\Re[\bar{d}]$ del argumento válido e inválido respectivamente. En la primera expresión "d" es un término general para lo divino y en la última " $d$ " para lo no-divino - lo que los escolásticos llamaban un "término infinito" (los lógicos de hoy hablan del "complemento" de D). Debemos permitir la equivalencia ("inf"):

$$
S_{i} /(T)<>S_{i} \bar{T}_{i}
$$

(donde $S$ y $\mathrm{T}$ son términos e i el índice). ${ }^{25}$ Un término infinito puede considerarse un nombre vago de cualquier individuo que no tiene la propiedad en cuestión. Por ejemplo, Brunelo (con el índice 7 digamos), sería llamado no solamente $j_{7}$ (aqueste jumento), $a_{7}$ (aquel animal), etc., sino también $\bar{d}_{7}$ (aquella cosa no-divina), dado que $b_{7} /(d), a_{7}$ (áquel no-árbol), etcétera. ${ }^{26}$ Una parte de la prueba extensional iría como sigue. Nota que el único análisis de $d$ es, digamos, $d_{d}$ :

\begin{tabular}{l|ll}
1 & $(h) /(d)$ & $\mathrm{h}$ \\
2 & $(j) \Re[h]$ & $\mathrm{h}$ \\
& & \\
3 & $h_{1} /(d) \& h_{2} /(d) \& \ldots \& h_{n} /(d)$ & dc 1 \\
4 & $h_{1} /(d)$ & ec 3 \\
5 & $h_{1} d_{1}$ & inf 4 \\
6 & $j_{7} \Re[h] \& j_{8} \Re[h] \& \ldots \& j_{h} \Re[h]$ & dc 2 \\
7 & $j_{7} \Re[h]$ & ec 6 \\
8 & $j_{7} \Re h_{1} v j_{7} \Re h_{2} v \ldots v j_{7} \Re h_{n}$ & dd 7 \\
& & \\
9 & $\mid j_{7} \Re h_{1}$ & h (de 8$)$ \\
& $\mid+h_{1} \bar{d}_{1}$ & r 5 \\
10 & $\mid h_{7} \bar{d}_{1}$ & i 9.10
\end{tabular}

25 Compárese con $\bar{T} x<>\sim T x$, donde $T$ es un predicado y $x$ una variable individual.

26 Véase Redmond, "Extensional...", pp. 45-46. 
12||$j_{7} \Re d_{1} v j_{7} \Re \bar{d}_{2} v \ldots v j_{7} \Re \bar{d}_{n} \quad$ id 11

13||$j_{7} \Re[\bar{d}]$ ad 12

$k \mid j_{7} \Re[\bar{d}]$

de $8,9-13 \ldots$

$k+1 \mid j_{8} \Re[\bar{d}]$

$\ldots$ de

$k+2 \mid j_{h} \Re[\bar{d}]$

$\ldots$ de

$k+3 \mid j_{7} \Re\left[\bar{d} \& j_{8} \Re[\bar{d}] \& \ldots \& j_{h} \Re[\bar{d}] \quad\right.$ ic $k, k+1, k+2$

$k+4 \mid(j) \Re[\bar{d}]$

ac $k+3$

Alonso, como hemos visto, ofrece ejemplos de términos oblicuos en casos diferentes del genitivo. Aquí hay otro ejemplo $(116 \mathrm{~B})$ del dativo (homini, "para un hombre"):

omni homini convenit appetitum subjicere spiritui

juvenis est homo

ergo

juveni convenit appetitum subjicere spiritui

(para cada hombre es menester sujetar las pasiones al espíritu/ un joven es un hombre/ luego: para un joven es menester sujetar las pasiones al espíritu). El siguiente argumento tiene en acusativo (homines - juzgar"seres humanos"):

omnes homines judicabit Deus

potentes saeculi sunt homines

ergo

potentes judicabit Deus

(Dios juzgará a todos los hombres/ los poderosos del mundo son hombres/ luego: Dios juzgará a los poderosos). ${ }^{27}$

\section{Más de tres términos}

La regla general de que un silogismo debería tener solamente tres términos $\mathrm{y}$ tres oraciones, dice Alonso (96 B), ha de entenderse de sus

27 Claro que podemos interpretar estos ejemplos como silogismos sencillos o como argumentos donde se expresa una relación. Por ejemplo, s: "lo que sujeta las pasiones al espíritu" y $\Re$ "aquel para el cual es menester...", y $\mathrm{m}(\mathrm{y} \Re \mathrm{s}$ ): "aquel a quien es menester sujetar las pasiones al espíritu". También, d: "Dios" y "que... júzgará" y j (y $\Re$ d): "que Dios juzgará".

$\begin{array}{llll}\mid(h) \Re[s] & \mid(h)[m] & \mid(h) \Re d & \mid(h)[j] \\ \mid[j][h] & \mid[j][h] & \mid(p)[h] & \mid[p][h] \\ \vdash & \vdash & \vdash & \vdash \\ \mid[j] \Re[s] & \mid[j][m] & \mid(p) \Re d & \mid(p)[j]\end{array}$


partes esenciales sin las cuales no se puede formar un silogismo; pero no se sigue que no pueda tener más términos y oraciones, en tanto los términos estén debidamente dispuestos y las oraciones bien ordenadas. ${ }^{28}$

Puede ser una concatenación de oraciones (un sorites) o puede contener oraciones que exhiban esquemas más complejos que el de sujeto-predicado. Alonso da (97 A-B) cuatro "condiciones" para los silogismos y todos los ejemplos que ofrece para ilustrarlas tienen más de tres términos. Considermos primero estos ejemplos (4.1.) y luego las condiciones (4.2.).

\subsection{Ejemplos y análisis}

\subsection{Reír y relinchar}

Alonso ofrece dos ejemplos de argumentos con más de tres términos. El primero (96 B) tiene seis términos:

omnis homo est risibilis et omnis equus est hinnibilis

Petrus est homo et Brunnellus est equus

ergo

Petrus est risibilis et Brunellus est hinnibilis

(todo hombre es capaz de reír y todo caballo de relinchar/ Pedro es hombre y Brunelo es caballo/ luego: Pedro es capaz de reír y Brunelo de relinchar). El argumento dice (96 B), es "virtualmente dos silogismos", según el esquema del modo darii ${ }^{29}$ (que en el análisis están en los segmentos 3-5 y 6-8):

\begin{tabular}{|c|c|}
\hline $1 \mid(h)[r] \& \mathcal{C}[r]$ & $\mathrm{h}$ \\
\hline$\left.2\right|_{\vdash} p[h] \& b[c]$ & $\mathrm{h}$ \\
\hline $3 \mid(h)[r]$ & ec 1 \\
\hline $4 \mid p[h]$ & ec 2 \\
\hline $5 \mid p[r]$ & darii 3,4 \\
\hline $6 \mid(c)[r]$ & ec 1 \\
\hline $7 \mid b[c]$ & ec 2 \\
\hline $8 \mid b[r]$ & darii 6,7 \\
\hline
\end{tabular}

28 ex partibus essentialibus. Itaque sine illis non potest fieri syllogismus. Sed tamen non sequitur ex hoc quod non possit habere plures terminos et plures propositiones, dummodo sint debite dispositi termini et bene ordinatae propositiones.

${ }^{29} \mathrm{El}$ modo darii: $(\mathrm{M})[\mathrm{J}] /[\mathrm{N}][\mathrm{M}] /$ luego: $[\mathrm{N}][\mathrm{J}]$. Aquí el término menor es singular. 

$9 \mid p[r] \& b[r]$
ic 5,8

\subsection{Caballos corriendo}

El segundo ejemplo, dice, contiene básicamente tres términos:

cujuslibet hominis albi equus currit

Petrus vel Paulus est homo albus

ergo

Petri vel Pauli equus currit

(de todo hombre blanco corre un caballo/ Pedro o Pablo es un hombre blanco/ luego: de Pedro o de Pablo corre un caballo).

\subsection{Análisis actual}

Veamos primero cómo puede analizarse el argumento en la lógica actual. Las oraciones podrían traducirse así (u: "Pablo", T: "correr"):

| $U x[[H x \& B x]>E y[C y \& P x y \& T y]]$

| $[H p \& B p] v[H u \& B u]$

$\vdash$

...

| Ey[Cy \& Ppy \& Ty] v Ey[Cy \& Puy \& Ty]

Pero como las dos conjunciones $H x \& B x$ y Ey[Hy \& Pxy \& Ty] difieren solamente en $x$ y sus instancias $p$ y $u$ ( $c f r$. la simplificación en 3.11.), podemos reescribirlas respectivamente como $\phi x$ (ser hombre blanco) y $\Theta x$ (ser propietario de un caballo corredor) y obtenemos esta prueba:
$1 \mid U x[\phi x>\theta x]$
$\mathrm{h}$
$2 \mid \phi p v \phi u$
h
1
$3 \mid \phi p>\Theta p$
ecu $1, p / x$
$4 \mid \phi u>\theta u$
ecu $1, u / x$
5||$\phi p$
h (de 2)
6||$\phi p>\theta p$
r 3
7||$\Theta p$
mp 5, 6
8||$\theta p v \theta u$
id 7
$\left.9\right|_{\vdash} ^{\mid \phi u}$
h (de 2) 


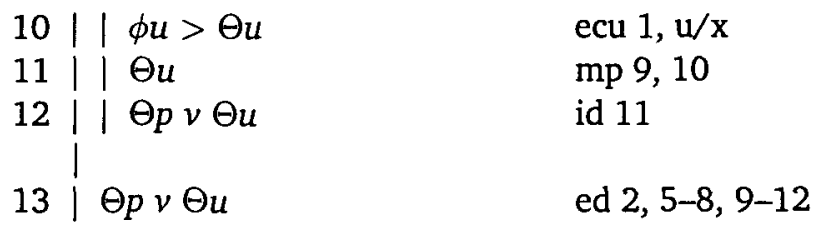

La segunda premisa y la conclusión son oraciones moleculares (disyunciones) y los tres términos de los que habla a Alonso son $\phi, \Theta$ y $x$ (más sus instancias $p \mathrm{y} u$ ).

\subsection{Análisis extensional}

Presuponiendo la doctrina sobre las unidades complejas ( $v$ y $\&$ son los juntores " $o$ " e " $y$ "), ${ }^{30}$ podemos representar extensionalmente el argumento así:

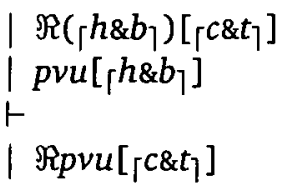

Nota que las frases "hombre blanco" y "caballo corredor" se interpretan como unidades complejas "complexivas de sentido singular" y que funcionan como términos vagos; además la segunda premisa consta de dos unidades complejas, la primera dividida y la segunda complexiva, y su análisis va así:

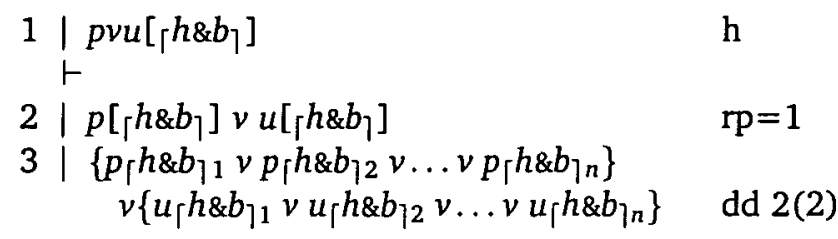

(paso 3: Pedro es este hombre blanco o ese hombre blanco o... aqueste hombre blanco, o Pablo es este hombre blanco o ese hombre blanco... o aqueste hombre blanco).

Como para los propósitos de la inferencia no necesitamos analizar las unidades ${ }_{\lceil} h \& b_{\rceil} y_{\lceil} c \& t_{\rceil}$, pues no muestran variaciones internas, las reemplazmos por los símbolos $\Phi$ ("ser humano y blanco") y $\Psi$ ("caballo corredor" -aquí no $\Theta$ : "lo que posee a un caballo corredor"). Alonso dice que el silogismo está en darii y que incluso cuando se reformula de manera simplificada aparece como una variante de ese modo (aunque el término menor es dividido más bien que cuantificado):

$1 \mid \Re(\phi)[\Psi]$

30 Redmond, "La lógica de. . " y "Relations. .. ", 24-25, 33; \&/v en lugar de $c / d$. 


\section{$2 \mid p v u[\phi]$ \\ 1 \\ 3 | $\Re p v u[\Psi]$.}

La prueba de identidad va así:

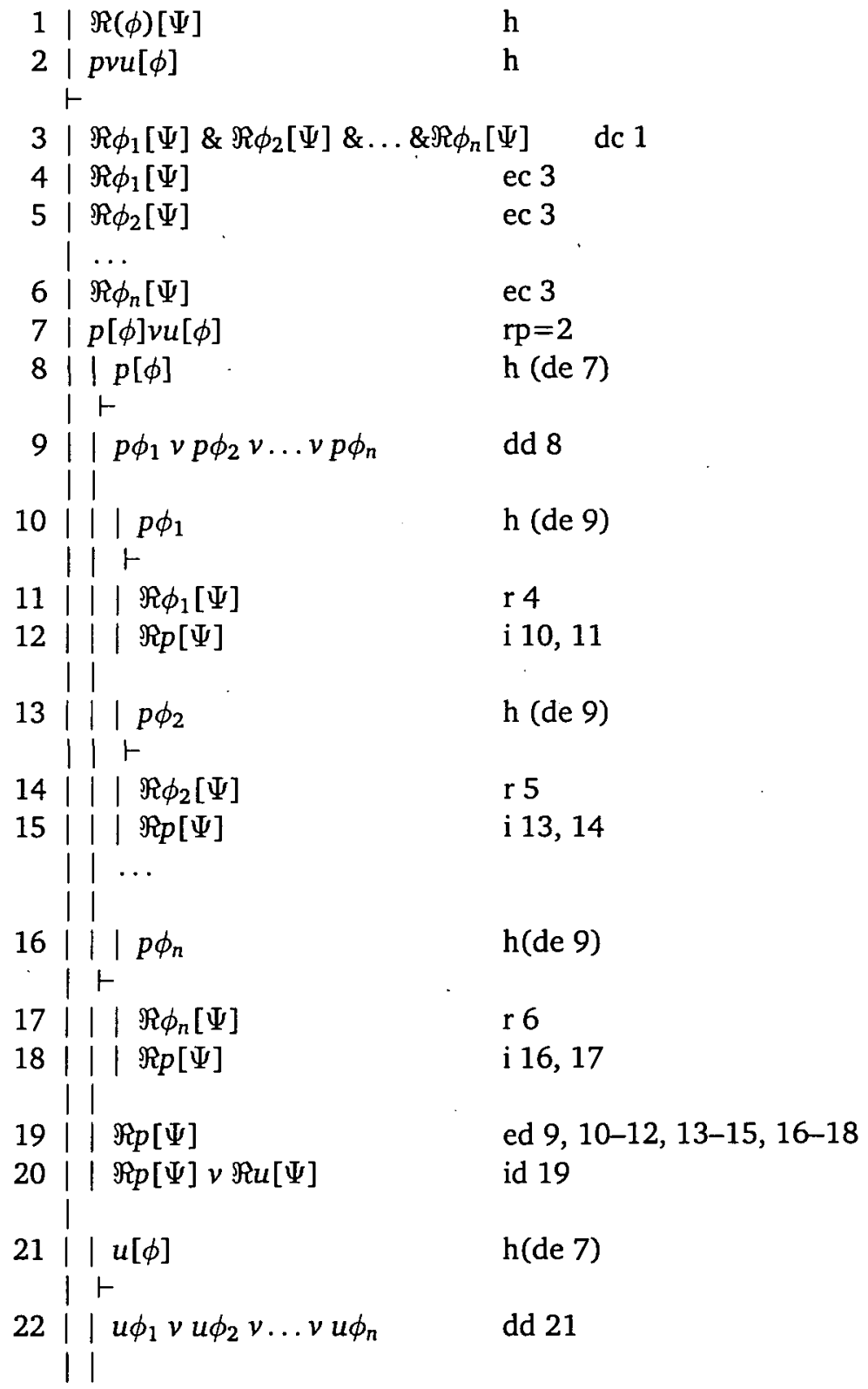




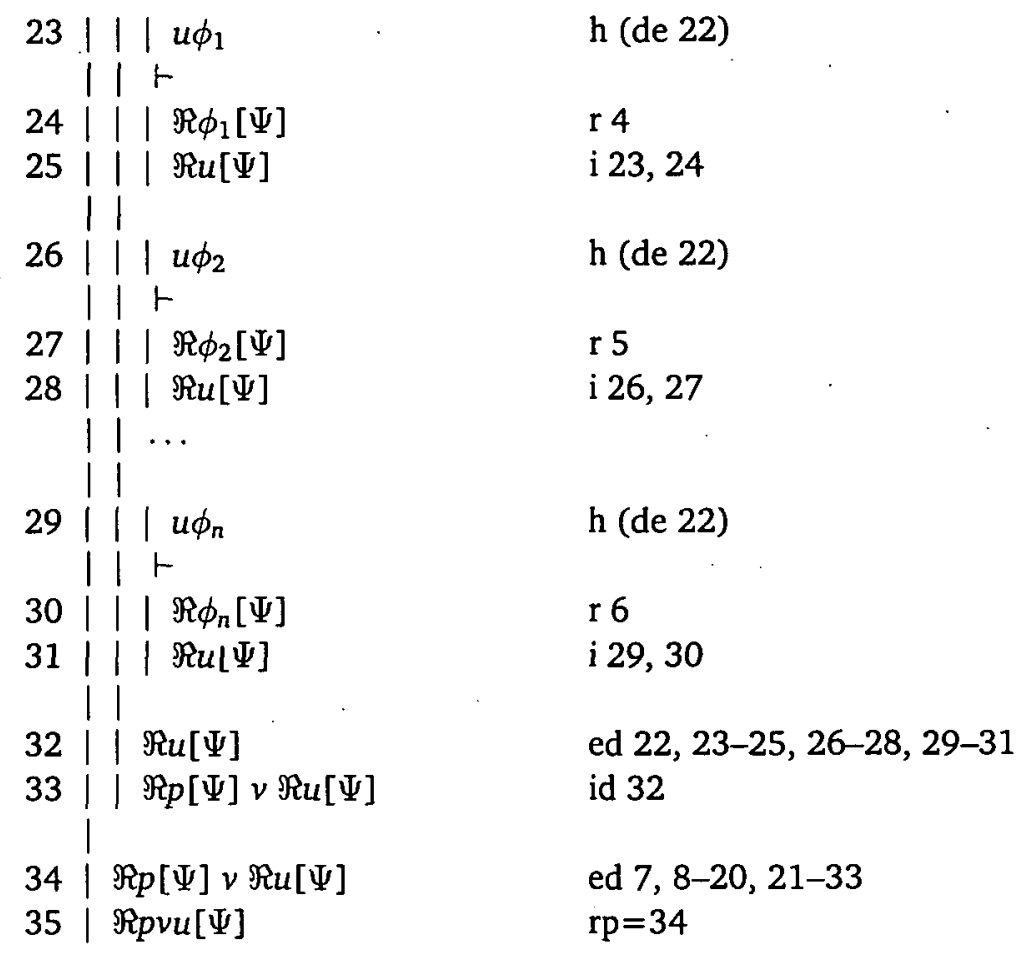

El argumento es un doble silogismo, porque la segunda premisa y la conclusión son disyunciones virtuales donde cada parte $(1,8-19$ y 1, 21-32) se asemejan al argumento de 2.1. También los pasos donde se aplica la regla-i (10-12, etcétera), pueden compararse con la prueba en el esquema normal de darii (a la derecha):

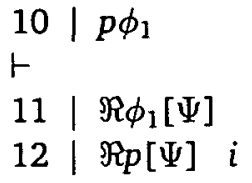

4.2. Condiciones

Al final del primer capítulo ( 97 A) Alonso pone, rápidamente,

cuatro condiciones que se requieren para ordenar debidamente las premisas entre ellas y en relación con la conclusión ${ }^{31}$

31 Ad debitam praemissarum dispositionem tam inter se quam in ordine ad conclusionem, quatuor conditions sunt requisitae. 
Los silogismos que ofrece como ejemplos contienen más de tres términos y las "condiciones parecen incluso extender las reglas de los silogismos simples a casos más complejos.

\subsection{La primera condición}

Sin embargo, no parece restringir la primera condición, que dice:

el término medio debería ser una unidad completa en la otra premisa. ${ }^{32}$

Las premisas del siguiente argumento, arguye, están ordenadas incorrectamente:

cujuslibet hominis quilibet equus currit

cujuslibet hominis equus quiescit

ergo

equus currit et quiescit

(de todo hombre todo caballo corre/ de todo hombre un caballo descansa (d)/ luego: un caballo corre y descansa), dado que

la expresión "de todo hombre" considerada como término medio no es una unidad completa en la otra premisa. ${ }^{33}$

En el caso de más de dos términos el signo relacional $\Re$ tiene el efecto de unir los dos primeros términos, y la conclusión contiene una unidad "complexiva, de sentido singular": ${ }^{34}$

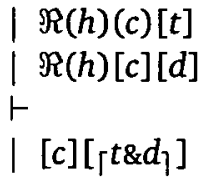

Si $T$ y $D$ son inconsistentes, las premisas de la siguiente prueba actual implican una contradicción; pero la prueba es válida (hay que agregar la primera premisa para expresar la aplicación existencial, tácitamente presupuesta por Alonso):

32 Medium debet esse totale extremum in altera praemissarum.

33 ly cujuslibet hominis quod assumitur pro medio non est totale extremum in altera praemissarum.

34 Nota la convención del orden, Redmond, "Extensional...", pp. 51, 64-65; Alonso considera $T$ y $D$ inconsistentes; el último ejemplo de la sección $(79 \mathrm{~B})$ tiene: "dos caballos: uno corre y el otro no". Para la conclusión véase Redmond "Relations... "; las premisas también podrían expresarse como unidades complejas. 


\begin{tabular}{|c|c|c|}
\hline 1 & $E x H x$ & $\mathrm{~h}$ \\
\hline 2 & $U x[H x>U y[[C y \& P x y]>T y]]]$ & h \\
\hline 3 & $U x[H x>E y[C y \& P x y \& D y]]$ & $\mathrm{h}$ \\
\hline 4 & $x \underset{\vdash}{\mid} H x$ & $\mathrm{~h}$ \\
\hline 5 & $\mid H x>U y[[C y \& P x y]>T y]$ & r 2 , ecu \\
\hline 6 & $U y[[C y \& P x y]>T y]$ & $\operatorname{mp} 4,5$ \\
\hline 7 & $H x>E y[C y \& P x y \& D y]]$ & r 3, ecu \\
\hline 8 & $E y[C y \& P x y \& D y]]$ & $\operatorname{mp} 4,7$ \\
\hline 9 & $\mid \begin{array}{l}y \mid C y \& \text { Pxy \& Dy]] } \\
\mid\end{array}$ & $\mathrm{h}$ \\
\hline 10 & $\mid C y \& P x y$ & ec 9 \\
\hline 11 & $\mid[C y \& P x y]>T y$ & r $6, \mathrm{ecu}$ \\
\hline 12 & | Ty & $\mathrm{mp} 10,11$ \\
\hline 13 & | Cy \& Ty \& Dy & ic 10,12 \\
\hline 14 & | Ey $[C y \& T y \& D y]$ & ice 13 \\
\hline 15 & $E y[C y \& T y \& D y]$ & ece $8,9-14$ \\
\hline 16 & $E y[C y \& T y \& \dot{D} y]$ & ece $1,4-15$ \\
\hline
\end{tabular}

En el argumento extensional el segmento en el término medio que acompaña a (h) es diferente en cada premisa: " $\ldots$. . (c)" y “ $\Re$. . [c]". La situación es realmente compleja: la combinación de cuantificadores en el segmento afectado por la relación $(\Re)$ habrá determinado la validez de dichos argumentos. ${ }^{35}$

Por otra parte, el segundo ejemplo ${ }^{36}$ de Alonso, donde el segmento “R...[a]" no cambia:

cujuslibet hominis animal est album

hominis animal est nigrum

ergo

animal nigrum est album

(de todo hombre un animal es blanco/ de algún hombre un animal es negro/ luego: un animal negro es blanco)

$1 \mid \Re(h)[a][b]$

$2 \mid \Re[h][a][n]$

35 Siete formas son inválidas (donde las premisas contienen $[c] \ldots /[c] \ldots y / o[t] \ldots /[t]$; en las formas válidas debe expresarse la aplicación existencial (tres veces) ExCx y EyTy.

36 Instatur hic... 


\section{$3 \stackrel{\vdash}{\vdash}\left[a \& n_{\rceil}\right][b]$}

es un argumento inválido, puesto que el antecedente, explica Alonso, puede ser verdadero sin que lo sea la conclusión: cuando todo hombre tiene un animal blanco y alguno tiene también un caballo negro. ${ }^{37 .}$

\subsection{Segunda condición}

La segunda condición es:

el término medio, en tanto medio, no debería entrar en la conclusión, pero accidentalmente no es inconveniente, como cuando sirve para mantener la ampliación o la restricción. ${ }^{38}$

Alonso da por bueno este argumento:

Pater generat

Pater est essentia divina

ergo

essentia divina est Pater qui generat

(el Padre engendra (g)/ el Padre es la Esencia divina/ luego: la Esencia divina es el Padre que engendra), porque, dice, si el término medio "Padre" no formara parte de la conclusión, podríamos sacar la conclusión, teológicamente incorrecta:

essentia divina generat

(La Esencia divina engendra), y en este caso el argumento tendría antecedente verdadero y consecuente falso.

No obstante, el argumento inválido parece seguirse tanto en nuestros días como en la lógica extensional (e: "Esencia divina"):
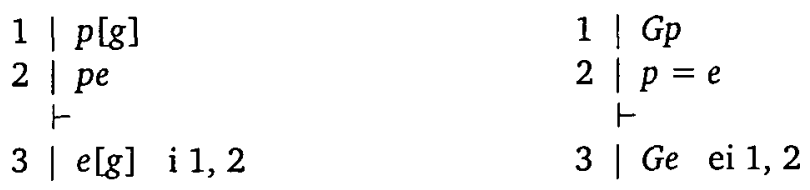

Alonso aceptaría - con algunas reservas- como ortodoxo el último paso de la siguiente prueba, que traduce "la Esencia divina es el Padre que engendra":

$37 U x[H x>E y[A y \& P x y \& B x]] \& E x[H x \& E y[A y \& P x y \& N x]]$ no implica $E y[A y \& N y$ $\& B y]$.

${ }^{38}$ Medium subratione medii non intret conclusionem, de per accidens tamen non inconvenit, ut si ponatur ad servandam ampliationem aut restrictionem. 


\begin{tabular}{l|l}
$1 \mid G p$ & $\mathrm{~h}$ \\
$2 \mid p=e$ & $\mathrm{~h}$ \\
& \\
$3 \mid$ & \\
$4 \mid p=p$ & ai \\
$5 \mid E x[x=e \& x=p \& G x]$ & ic $1,2,3$
\end{tabular}

puesto que admitiría —en algún sentido- que hay algo que es la Esencia divina, que es el Padre y que engendra. El fraile trata los complejos problemas de la identidad en un capítulo sobre "los términos divinos", donde elabora maneras de bloquear las inferencias heterodoxas y permitir las aceptables. ${ }^{39}$

De todos modos, agrega que "no sería correcto" que el término medio entrara en la conclusión "innecesariamente", ${ }^{40}$ como en:

omnis homo disputat

Petrus est homo

ergo

Petrus est homo qui disputat

sed... solum: Petrus disputat

(todo hombre disputa/ Pedro es hombre/ luego: Pedro es un hombre que disputa); sólo admitiría la conclusión de que Pedro disputa.

Es difícil ver por qué la primera conclusión ("Pedro es un hombre que disputa") "no es correcta". ¿Es falsa porque "que disputa", como "Que engendra", es una cláusula restrictiva "como medio"? Esto es, ¿Piensa él que implica que como Esencia divina, aunque idéntica al Padre que engendra, no engendre, de tal manera que es posible que Pedro, siendo idéntico a algún hombre que disputa, no dispute? En este caso debemos examinar más de cerca la doctrina sobre la identidad de Alonso y sus colegas.

\subsection{Tercera condición}

Antes de presentar la tercera condición, estudiemos los silogismos válidos que ofrece Alonso para explicar su importancia. El primer ejemplo ilustra la primera parte de la condición.

cujuslibet hominis equus currit

Petrus est homo

ergo

Petri equus currit

39 Redmond, "Friar Alonso on the Logic of God", Vivarium, xxxii, 1994, 2, pp. 227-260.

40 Absque necessitate non recte concluderetur... 
(de todo hombre un caballo corre/ Pedro es hombre/ luego: de Pedro un caballo corre), y si marcamos los términos, como quiere Alonso,
$1 \mid \Re(h)[c][t]$
$2 \mid p[h]$
$\vdash$
$3 \mid \Re p[c][t]$
$1 \mid \Re(M)[c][J]$
$2 \mid N[M]$
$\vdash$
3 | $\Re N[c][J]$

tenemos que el segmento " $\Re .$. [c]" no cambia. Esta simplicidad se refleja en una versión actual ( $\phi x$ representa $E y[C y \& P x y \& T y])$ :
$1 \mid U x[H x x>\phi x]$
$\mathrm{h}$
$2 \mid H p$
$\mathrm{h}$
$\vdash$
$3 \mid H p>\phi p$
ecu $1 p / x$
$4 \mid \phi p$
mp 2, 3

Su segundo ejemplo, que corresponde a la segunda parte de la condición, sigue el mismo esquema:

omnis homo est animal

Brunellus est hominis equus

ergo

Brunellus est animalis equus

(todo hombre es animal/ Brunelo es caballo de un hombre/ luego: Brunelo es caballo de un animal).
$1 \mid(h)[a]$
$2 \mid b \Re[h][c]$
$1 \mid(M)[J]$
$\vdash$
$3 \mid b \Re[a][c]$
$2 \mid N \Re[M][c]$
$\vdash$
$3 \mid N \Re[J][c]$

Ni el segmento " $\Re$... [c]" alterado aquí ni su prueba, donde $\Theta$ representa $[b=y \& C y]$, son sencillos:

| $U x[H x>A x]$

| Ey $\Theta y \& E x[H x \& P x y]]$

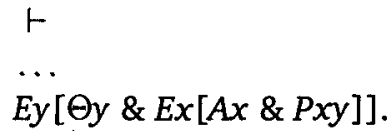

He aquí una traducción — reordenada un poco— de la tercera condición: 
la conclusión [paso 3], y este término en la conclusión ["ßN [c]"] ha de ser como el término medio en la premisa mayor [" $\Re(M)[c]$ "].

[2 - corresponde al segundo ejemplo] y lo que afecta al término medio en la premisa menor [paso 2; i.e., el segmento "...$[c]$ "] ha de aplicarse al término mayor en la conclusión, y éste en la conclusión ["গR[J][c]"] ha de ser como el término medio en la premisa menor [" $R[M][c]$ "] ${ }^{41}$

\subsection{Cuarta condición}

La cuarta condición afecta la "totalidad" de los términos mayor y menor. Alonso usa (97 B) este silogismo es inválido para mostrar cómo no se cumple con la cuarta condición:

cujuslibet hominis equus currit

risibilis equus non currit

ergo

risibile non est homo

(de todo hombre un caballo corre/ de algo capaz de reír un caballo no corre/ luego: algo capaz de reír no es un hombre). Podría representarse así:

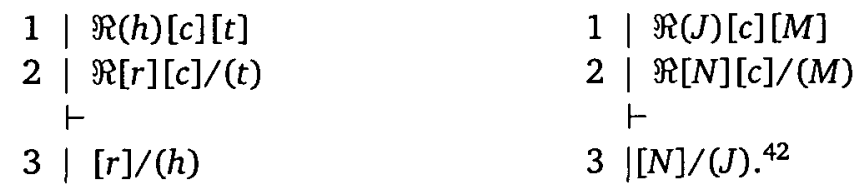

La condición va así:

los términos mayor y menor han de ser unidades completas tanto en las premisas como en la conclusión, a menos que:

algo se añada para mantener la corrección lógica o que una añadidura acompañe al término medio que ha de afectar a los términos mayor y menor [en la conclusión] para mantener la tercera condición. ${ }^{43}$

${ }^{41}$ Id quod se tenet ex parte medii in majori debet se tenere ex parte minoris extremitatis in conclusione, et quod se tenet ex parte medii in minori debet aplicari majori extremitati in conclusione, et debet concludi minor extremitas in eodem casu quo sumitur medium in majori et major extremitas in eodem casu quo stat medium in minori.

$42 U x\left[H x>E y\left[C y \& P x y \& C^{\prime} y\right]\right] \& E x\left[R x \& E y\left[C y \&\right.\right.$ \&xy \& $\left.\left.\sim C^{\prime} y\right]\right]$ no implica $\operatorname{Ex}[R x \& \sim H x]$.

43 Extremitates debent esse totalia extrema, tam in praemissis quam in conclusione, nisi id ponatur ad servandam logicalem proprietatem vel aliquid ponatur cum medio quod debeat applicari extremitatibus, juxta praedicta in tertia conditione. 
Los términos mayor y menor del silogismo no están completos en las premisas y en la conclusión, aparentemente porque en las premisas incluyen una añadidura (" $\Re[c]$ ") que no está en la conclusión. Específicamente, el segmento evidentemente no se aplica al término medio, y Alonso explica la falla de esta manera:

Si los sujetos completos de las premisas son el término mayor ["ß $(J)[c]$ "] y menor [" $R[N][C]$ "], no son unidades completas en la conclusión ["[N] y $(J)$ "], y si son las únicas determinaciones [en la conclusión], no son términos completos en las premisas. ${ }^{44}$

Añade, en la última edición, que el antecedente del argumento es verdadero y el consecuente falso en el caso de que todo hombre tenga dos caballos, uno que corre y otro que no.

\section{Semántica}

Examinaremos ahora, brevemente, la semántica de los términos oblicuos, considerando la "semántica" como la relación que existe entre los términos en la oración y aquello a lo que se refieren o significan.

Una comparación entre los pasos 8 y 9 del silogismo sencillo en 2.2. y los mismos pasos del silogismo oblicuo en 3.11. (los pasos a los que se aplica la regla-i) revelan una diferencia semántica. En el primer argumento, el paso 8 es una identidad donde los dos términos $\left(p\right.$ y $\left.h_{1}\right)$ se refieren a ("suponen por", como dirían los escolásticos) la misma cosa (Pedro). De aquí que $h_{1}$ pueda reemplazar a $p$ en el paso 10 . Suponiendo que el disyunto verdadero de $p[a]$ es $p a_{1}$, los términos $p$ y $a_{1}$ obviamente ambos se refieren a Pedro.

El caso del silogismo oblicuo (3.22.) es diferente $¿$ Digamos que $\Re h_{1} b_{5}$ es el disyunto verdadero de 9 y $\Re p b_{5}$ el disyunto verdadero de 10 , y además que $b_{5}$ se refiere a Platero, el burro que pertenece a Pedro. El paso 8 es una identidad sencilla $\left(p h_{1}\right)$, así que la sustitución en 9 nos puede llevar a $\Re p b_{5}(\Re p[b])$. A pesar de tener índices diferentes (1 y 5 ) los términos de 9 y $10\left(\Re h_{1}, b_{5}\right.$ y $\Re p$ ) se refieren solamente a Platero (no a Pedro y Platero). Puesto que Platero tiene la propiedad $B$ (ser burro), tiene el nombre (término vago) $b_{5}$, y puesto que tiene la relación $\Re$ con "este hombre", $h_{1}$ (Pedro), tiene el nombre $\Re h_{1}$, que puede considerarse aquí como un nombre propio (o descripción): "la propiedad de este hombre", indicando a Pedro. El nombre propio $\Re h_{1}$ es de tipo especial, puesto que contiene un térmi-

44 nam si totalia subjecta sumantur in praemissis pro extremitatibus, non sunt totalia extrema in conclusione, et si solae determinationes, non sunt totalia extrema in praemissis. 
no vago $\left(h_{1}\right)$ y el nombre propio $\Re p[b]$ contiene un término cuantificado ([b]).

Consideremos también el argumento que contiene un término infinito ( $\bar{d}$ : "no-divino" en 3.222. Las expresiones " $R[\bar{d}]$ " y " $\Re \bar{d}_{2}$ " pueden igualmente considerarse como nombres propios de un tipo especial puesto que contienen un término cuantificado ( $[\bar{d}])$ o un término vago $\left(\bar{d}_{2}\right)$. Nota que los índices en el paso 11 " $j_{7} \Re \bar{d}_{1}$ " obviamente no se refieren a la misma cosa; $j_{7}$ se refiere a "aquesta bestia de carga" (Brunelo) y $\bar{d}_{1}$ a "este hombre" $\left(h_{1}\right)$. Sin embargo, las unidades que constituyen la oración -el término vago $j_{7}$ ("aqueste jumento") y el nombre propio $\Re \bar{d}_{2}$ ("la propiedad de este hombre")- se refieren a la misma cosa: Brunelo. Así que $b_{7} \Re \bar{d}_{1}$ puede leerse: "aqueste jumento es propiedad de este hombre".

\section{AbreVIATURAs}

ac: ascenso conjuntivo

ad: ascenso disyuntivo

ai: axioma de la identidad

dc: descenso conjuntivo

dcu: descenso conjuntivo en unidad complexiva

$\mathrm{dd}$ : descenso disyuntivo

dt: distribución en unidad complexiva

ec: eliminación de la conjunción

ece: eliminación del cuantificador existencial

ecu: eliminación del cuantificador universal

ed: eliminación de la disyunción

ei: eliminación de la identidad

en: eliminación de la negación

h: hipótesis (premisa)

i: identidad (la regla-i)

ic: introducción de la conjunción

ice: introducción del cuantificador existencial

icu: introducción del cuantificador universal

id: introducción de la disyunción

ii: introducción de la implicación

in: introducción de la negación

inf: equivalencia de la negación y un término infinito

$\mathrm{J}$ : término mayor (con el término medio en la segunda premisa) ${ }^{45}$

M: término medio (presente en ambas premisas)

45 Los términos medio, mayor y menor se definen de acuerdo con Pedro Hispano. 
mp: modus ponens

mt: modus tollens

$\mathrm{N}$ : término menor (con el término medio en la segunda premisa)

$\mathrm{r}$ : reiteración (dentro de una subprueba)

$\mathrm{rp}=$ : definición de "sentido dividido" (repetición)

Traducido del inglés por: Juan Manuel Campos Benttez 